\title{
Human Factor Considered Risk Assessment of Automated Vehicle Using Vehicle to Vehicle Wireless Communication
}

\author{
Donghoon Shin ${ }^{1)}$ Kyongsu $\mathbf{Y i}^{2)}$ \\ 1) Republic of Korea Naval Academy, Department of Mechanical Engineering \\ 1, Jungwon-ro, Jinhae-gu, Changwon-si, Gyeongsangnam-do, \\ Korea 88-1-2, 51704, Sohn Won Yil-gwan \#205 \\ 2) Seoul National University, Department of Mechanical and Aerospace Engineering \\ 301-dong 113-ho, 1 Gwanak-ro, Gwanak-gu, Seoul 08826, Korea (E-mail: kyi@,snu.ac.kr)
}

Received on October 2, 2017

\begin{abstract}
This paper describes a human factor considered risk assessment of automated vehicle using vehicle to vehicle wireless communication (V2V communication). A human-reaction time is incorporated into the probabilistic threat assessment algorithm for the human-centered risk assessment. The V2V communication have been fused with a radar sensor to achieve more enhanced tracking performance of automated vehicle. Information fusion of two tracks, V2V communication and radar sensor, is performed using Global Nearest Neighborhood (GNN) approach. A prediction of vehicle's motion follows the basic idea of the particle filtering. Based on the predicted behavior of vehicles, a collision risk is computed numerically and 321 driver data based human reaction time are incorporated to determine an active safety control intervention moment. The humancentered risk assessment algorithm has been applied to a collision avoidance scenario to monitor threat vehicles ahead and to find the best intervention point. Effects of the vehicular communication on a target vehicle state estimation and a vehicle safety control performance are investigated. The performance of the proposed algorithm has been investigated via computer simulation studies. It has been shown from both simulations and vehicle tests that the proposed human-centered risk assessment algorithm with the $\mathrm{V} 2 \mathrm{~V}$ communication can be beneficial to active safety systems in decision of controller intervention moment and in control of automated drive for the guaranteed safety.
\end{abstract}

KEY WORDS: safety, Risk assessment, vehicular communication, human factor [C1]

\section{Introduction}

To increase traffic safety and driver comfort, the area of active safety has dramatically evolved in the automotive industry during the last decades. Advanced Driver Assistance Systems (ADAS) such as Adaptive Cruise Control (ACC), Lane Keeping Assistant System (LKAS), and autonomous emergency brake (AEB) have been shown to have a positive impact on traffic safety. According to Shin et al ${ }^{(1)}$. it is expected that further developed ADAS and eventually highly automated driving will continue to increase traffic safety by compensating human errors.

As is known to all, automotive radar is the best fitted vehicular sensing technology for the target state estimation problem. However, in an application of automotive radars, one of the most significant problems is an uncertain measurement model problem which is wandering on the target's physical boundary. To cope with this problem, more accurate target vehicle's information such as yaw rate, absolute velocity and acceleration needs to be transmitted using the V2V communications as reported by Shin et al. ${ }^{(2),(9)}$.

$\mathrm{V} 2 \mathrm{~V}$ communication is considered since it allows the enhancement of already existing ADAS or Automated Drive system. Once the target vehicle information is transmitted to egovehicle, it can be used to improve safety, efficiency and ride comfort by enhancing perception or object vehicle detection performance. With accurate target detection and ranging performance, highly automated driving can be achieved by intervention of active safety for whole speed range. In other words, drivers are not expected to watch the system at all times while automated driving. This paper considers a human-centered approach to find the guaranteed margin of drive active safety.

In order to express the guaranteed margin of active safety mode, a collision risk and a human reaction time are considered to find intervention margin of the system. And this allows the humanlike naturalistic behavior of self-driving car which is safe and acceptable system from the drivers as well as other road users ${ }^{(3)}$.

The current stages of risk metrics are mainly based on the predicted time when some predefined risky event occurs. The typical predicted time indices include the time to collision or time to impact. According to a number of literature reviews, an assessment of collision risk which corresponds with driver behavior which also can be applied to many complex situations is discussed. A proposed risk assessment algorithm, in this paper, cover a range of all requirements by focusing on collision probability ${ }^{(4),(5)}$.

The paper is organized as follows. In section 2 , the information fusion of $\mathrm{V} 2 \mathrm{~V}$ communication and Radar is discussed. Section 3 describes human-centered risk assessment algorithm. The proposed human-centered risk assessment algorithm with the $\mathrm{V} 2 \mathrm{~V}$ 


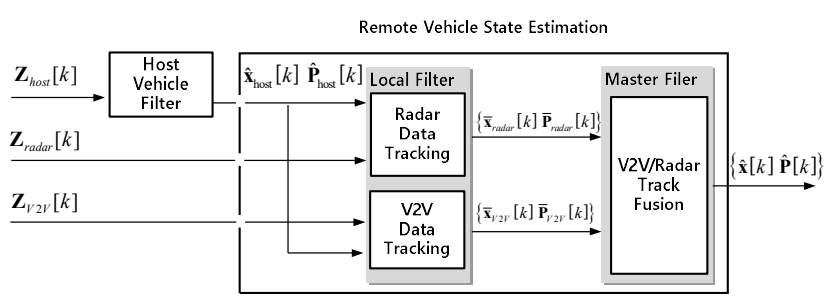

Fig.1 Information Fusion Architecture

communication is evaluated and validated via simulations in Section 4. Finally, conclusions are provided in Section 5.

\section{Information Fusion of V2V Communication and Radar}

The proposed high-level fusion architecture for the ADAS application consists of two main levels of sensor data processing: local filter, master filter illustrated in Fig.1.

In the radar data tracking local filter, an interacting multiple model approach using extended Kalman filters (EKF) was employed to estimate the remote vehicles' states such as heading, yaw rate, absolute velocity and acceleration as well as relative position and velocity. Data tracking of V2V communication is performed by implementing a state augmented estimation algorithm in order to compensate any possible latencies caused by vehicular communication. To describe the behavior of the remote vehicle, a kinematic vehicle model is derived. And a standard measurement model is defined to express the remote vehicle's CAN bus which transmits the vehicle information to the host vehicle. The state vector of $\mathrm{V} 2 \mathrm{~V}$ communication is defined as follows:

$$
\mathbf{x}_{r v}^{v 2 v}=\left[\begin{array}{lllllll}
p_{r, x}^{v 2 v} & p_{r, v}^{v 2 v} & \theta_{r v}^{v 2 v} & v_{r, x}^{v 2 v} & \gamma_{r v}^{v 2 v} & a_{r, x}^{v 2 v} & \dot{\gamma}_{v}^{v 2 v}
\end{array}\right]^{T}
$$

The EKF is used to estimate host vehicle states such as longitudinal velocity and yaw rate. The input signals, measurements, are the vehicle sensor signals such as wheel speed, acceleration, and yaw rate of which each time derivative is considered. The procedure of track creation and deletion are demonstrated with s consolidated and non-consolidated tracks.

When two tracks are decided to be a form of the same target, then the next problem is to provide a single target track which has less uncertainty than that of individual tracks themselves. This is a track-to-track fusion problem.

\subsection{Track-to-Track Association}

First step is to dismiss those non-likely association pair. In order to do that a gating procedure is performed. The gating creates a region that the most likely combinations are include. Using Global Nearest Neighborhood (GNN) approach, pairing process is performed when only detections are closer to a track than its respective gate. The associations that do not fall within the gate are considered to be non-likely, so that dismissed. Gating is performed using a square approach (2) :

$$
\text { Gate }=K \sigma_{r}
$$

where $\sigma_{r}$ is the residual standard deviation and $K$ is a constant that was empirically chosen (according to ${ }^{(14)}$ typically it is chosen to be $K \geq 3$ ). After gating, association is performed using Euclidean normalized distance and a stability factor, giving less priority to less stable measures, as presented in (3) :

$d^{2}=\frac{\left(x_{i}-\bar{x}\right)^{2}}{\sigma_{x}^{2}}+\frac{\left(y_{i}-\bar{y}\right)^{2}}{\sigma_{x}^{2}}+\ln \left(\sigma_{x} \sigma_{y}\right)$

where $d$ is the distance between previous and presented tracks to be associated, and $\left(\sigma_{x}, \sigma_{y}\right)$ the appropriate values of covariance matrix of Kalman Filter.

\subsection{Track Creation and Deletion Logic}

Track creation and deletion procedure is based on the definition of two different kind of tracks: consolidated and nonconsolidated. First refers to those tracks that were confirmed by both sensors whether concurrently or in subsequent scans. Later refers to tracks detected by a single sensor thus it is not trustable enough since the other sensor have not corroborated it. So, it is important to define the policy for creation and detection as well as to define when a track is updated i.e. it is considered to have a match with the new detections. This policy was empirically obtained.

The use of consolidated and non-consolidated tracks helps the system to add reliability. Only consolidated tracks are considered trustable detections. Hence, only them are reported. This way false positives, mainly from the laser scanner, are discarded because detections that are not corroborated by the vision system are not reported. Furthermore, the use of both sensors, once the track is consolidated, to update the tracks, allows that once a pedestrian is detected it can be tracked, even if it is not detected by one of the subsystems, e.g. when it goes out of the camera field of view.

\section{Human-Centered Risk Assessment Algorithm}

The human-centered risk assessment algorithm continuously monitors the risk and determines an active safety control intervention moment if required. In order to find decision margin, a trade-off moment between the human and the system has to be determined and it guarantees human-like behavior of the system and increases driver acceptance of autonomous system. The performance of the automated system is defined by its probability of detection (probabilistic collision risk) and that of the human behavior which is based on own processing of the event (humanreaction time). For the human-centered risk assessment, reasonable estimation of predicted pose and motion of remote vehicle with good accuracy is required. With computation of collision probability, most probable predicted collision distance, $\boldsymbol{D} \boldsymbol{p}_{\max C \boldsymbol{p}}$, is derived accordingly. Meanwhile a human-reaction time is computed with respect to the $\boldsymbol{D} \boldsymbol{p}_{\max } \boldsymbol{p} \boldsymbol{p}$. The human-reaction time and the collision probability with respect to most probable predicted collision distance are computed real time in order to find the active safety control intervention margin.

\subsection{Vehicle States Predictions}


The Kalman filter is widely used to estimate the states represented in a state space equation. In order to establish a state prediction, the vehicle state estimation method such as longitudinal velocity, yaw rate, longitudinal acceleration and yaw acceleration has been developed. A road geometry estimation has been established with the measurements from a vision sensor. The road geometry can be described by a $2^{\text {nd }}$ order polynomial. Likewise with the vehicle states estimation, the Kalman filter is used for the estimation of road geometry coefficients of the polynomial. The state prediction of the vehicle has been established under the assumptions that a host and preceding vehicle maintain current behavior in the finite time horizon. The vehicle position in the finite time horizon is represented as follows :

$$
\begin{aligned}
\mathbf{x}_{p}[i+1]= & f_{p}\left(\mathbf{x}_{p}[i]\right)+\mathbf{w}_{p}[i], \\
= & {\left[\begin{array}{lllllll}
f_{1, p} & f_{2, p} & f_{3, p} & f_{4, p} & f_{5, p} & f_{6, p} & f_{7, p}
\end{array}\right]+. } \\
& \ldots+\mathbf{w}_{p}[i]
\end{aligned}
$$

where,

$$
\begin{aligned}
& \mathbf{x}_{p}=\left[\begin{array}{lllllll}
p_{x, p} & p_{y, p} & \theta_{p} & v_{p} & \gamma_{p} & a_{p} & \dot{\gamma}_{p}
\end{array}\right]^{T} \\
& f_{1, p}=p_{x, p}+\left(v_{p} \cos \theta_{p}\right) \Delta t+\left(a_{p} \cos \theta_{p}+\gamma_{p} v_{p} \sin \theta_{p}\right) \frac{\Delta t^{2}}{2} \\
& f_{2, p}=p_{y, p}+\left(v_{p} \sin \theta_{p}\right) \Delta t+\left(a_{p} \sin \theta_{p}+\gamma_{p} v_{p} \cos \theta_{p}\right) \frac{\Delta t^{2}}{2} \\
& f_{3, p}=\theta_{p}+\left(\gamma_{p}\right) \Delta t+\left(\dot{\gamma}_{p}\right) \frac{\Delta t^{2}}{2} \\
& f_{4, p}=v_{p}+\left(a_{p}\right) \Delta t+\left(-k_{a} a_{p}\right) \frac{\Delta t^{2}}{2} \\
& f_{5, p}=\gamma_{p}+\left(\dot{\gamma}_{p}\right) \Delta t+\left(-k_{\dot{\gamma}} \dot{\gamma}_{p}\right) \frac{\Delta t^{2}}{2} \\
& f_{6, p}=a_{p}+\left(-k_{a} a_{p}\right) \Delta t+\left(k_{a}^{2} a_{p}\right) \frac{\Delta t^{2}}{2} \\
& f_{7, p}=\dot{\gamma}_{p}+\left(-k_{\dot{\gamma}} \dot{\gamma}_{p}\right) \Delta t+\left(k_{\dot{\gamma}}^{2} \dot{\gamma}_{p}\right) \frac{\Delta t^{2}}{2} \\
& \mathbf{w}_{p}[i] \sim\left(0, \mathbf{W}_{p}[i]\right) \\
& \mathbf{W}_{p}[i]=\left(\mathbf{B}_{p} \Delta t+\mathbf{F}_{p}[i] \mathbf{B}_{p} \frac{\Delta t^{2}}{2}\right) Q_{p}\left(\mathbf{B}_{p} \Delta t+\mathbf{F}_{p}[i] \mathbf{B}_{p} \frac{\Delta t^{2}}{2}\right)^{T} \\
& \mathbf{F}_{p}[i]=\left.\frac{\partial f_{p}}{\partial x_{p}}\right|_{x_{p}=\hat{x}_{p}[i]} \quad \mathbf{B}_{p}=\left[\begin{array}{lllllll}
0 & 0 & 0 & 0 & 0 & 0 & 1 \\
0 & 0 & 0 & 0 & 0 & 1 & 0
\end{array}\right]^{T}
\end{aligned}
$$

The subscript ' $p$ ' in (8) denotes 'predictive'. A prediction procedure at the first future time step is illustrated in Fig.2. The desired yaw rate for lane keeping is supposed to be a virtual measurement as shown in the following equation.

$$
\begin{aligned}
& \mathbf{z}_{p}[i]=H_{p} \cdot \mathbf{x}_{p}[i]+\mathbf{v}_{p}[i], \quad i=0, \mathrm{~L}, N_{p} \\
& =\left[\begin{array}{lllllll}
0 & 0 & 0 & 0 & 1 & 0 & 0
\end{array}\right] \cdot \mathbf{x}_{p}[i]+\mathbf{v}_{p}[i] \\
& =\bar{\gamma}_{d e s, p}[i] \\
& =-C \cdot \overline{\mathbf{x}}_{e, p}[i]+\gamma_{f f, p}[i]
\end{aligned}
$$

where,

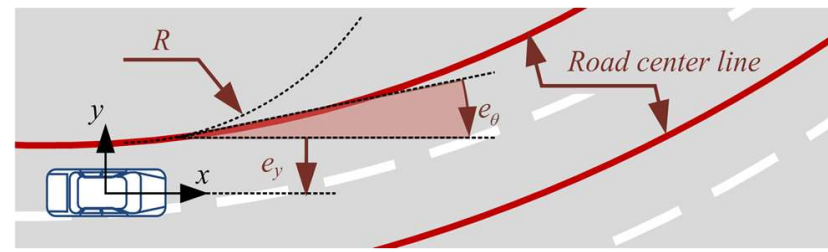

(a) The relationship between the host vehicle and the road center line of each lane

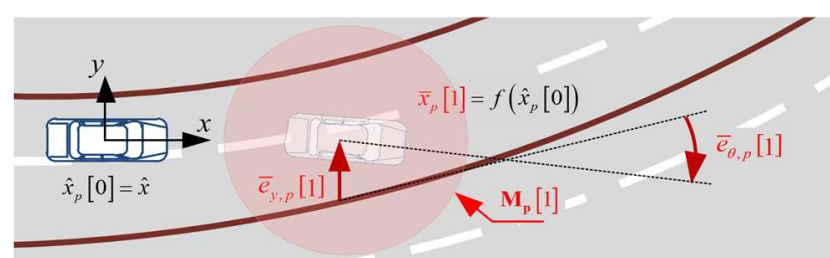

(b) The time-update-predicted host vehicle states and the relative error states with respect to road geometry defined on current body coordinate

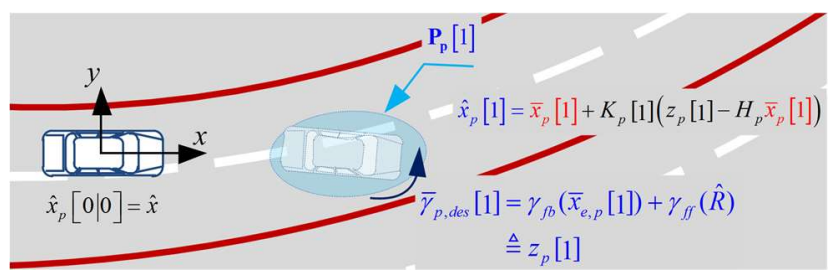

(c) The measurement-update-predicted host vehicle states where the predicted desired yaw-rate to keep the lane is defined as virtual measurement

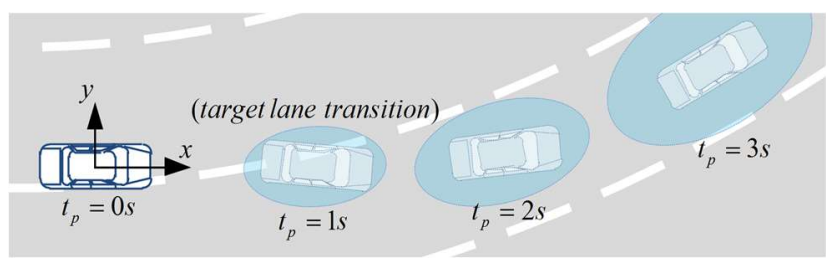

(d) Prediction results for $1 \mathrm{~s}, 2 \mathrm{~s}$, and $3 \mathrm{~s}$ of prediction time, $t_{\mathrm{p}}$, at a lane-changing instant

Fig.2 Procedure and Concept of Traffic Behavior Prediction

$$
\begin{aligned}
& \overline{\mathbf{x}}_{e, p}[i]=f_{e}\left(\overline{\mathbf{x}}_{p}[i], \hat{\mathbf{x}}_{r}[0]\right) \\
& =\left[\begin{array}{lll}
\bar{e}_{y}[i] & \bar{e}_{\theta}[i] & \bar{\gamma}_{p}[i]
\end{array}\right]^{T} \\
& =\left[\begin{array}{c}
\bar{p}_{y, p}[i]-\left\{\hat{a}_{2} \cdot \bar{p}_{x, p}[i]^{2}+\hat{a}_{1} \cdot \bar{p}_{x, p}[i]+\hat{a}_{0}+\hat{w}_{\text {road }} \cdot N[i]\right\} \\
\bar{\theta}_{p}[i]-\tan ^{-1}\left(2 \hat{a}_{2} \cdot \bar{p}_{x, p}[i]+\hat{a}_{1}\right) \\
\bar{\gamma}_{p}[i]
\end{array}\right] \\
& \mathbf{v}_{p}[i] \sim\left(0, \mathbf{V}_{p}[i]\right)
\end{aligned}
$$

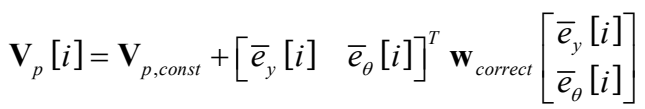

That is, behavior restrictions due to road geometry and driver characteristics such as reasonable acceleration input range are replaced with multi-stage of Kalman filtering problem by the proposed prediction algorithm. As an example, Fig.2-(b) and Fig.2(c) indicate a prediction procedure at the 1st future time step.. The overall prediction results for $1 \mathrm{~s}, 2 \mathrm{~s}$, and $3 \mathrm{~s}$ of prediction time at the instant of lane changing are expressed in Fig.2-(d). A detailed description of the vehicle's behavior prediction can be found in ${ }^{(6)}$. The ellipses around the vehicles in Fig.2 depict the reachable area of the vehicle. Since the proposed prediction algorithm is based on a stochastic filtering method, the covariance of the prediction error can be evaluated at each time step. 
Furthermore, the eigenvalue and eigenvectors of the 2nd leading principal minor of the covariance determine the likelihood ellipse around the predictive position. Using the square root of the eigenvalues as semi-axes measured along the eigenvectors, we can sketch the 39\% likelihood ellipse with the center at the most likely predictive position. This analysis is very useful to compute the probability of collision occurrence and assess the risk of current driving scene.

\subsection{Collision Probability}

With the prediction step stated in the previous section, the basic concept of the collision probability approximation has been described in Fig.3-(a). For each future time step, a mean and covariance matrix for the state of the vehicle are obtained to derive a mean trajectory with associated uncertainty (normal distribution at each time step).

We randomly generate a given number $\mathrm{N}$ state vectors based on the initial probability density function. is already introduced in the previous section. The parameter $\mathrm{N}$ could be chosen by the user as a trade-off between computational effort and collision probability

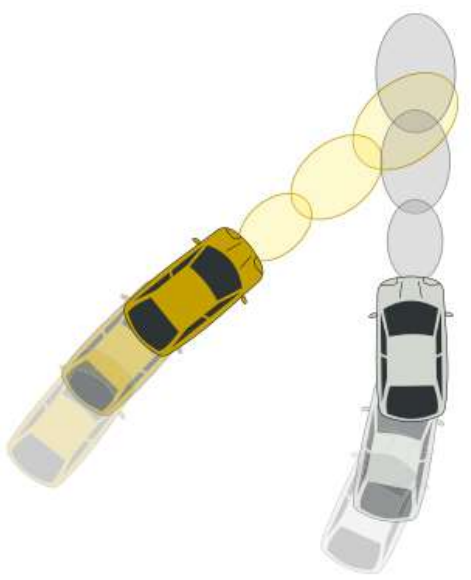

(a) Concept of Collision Probability Approximation

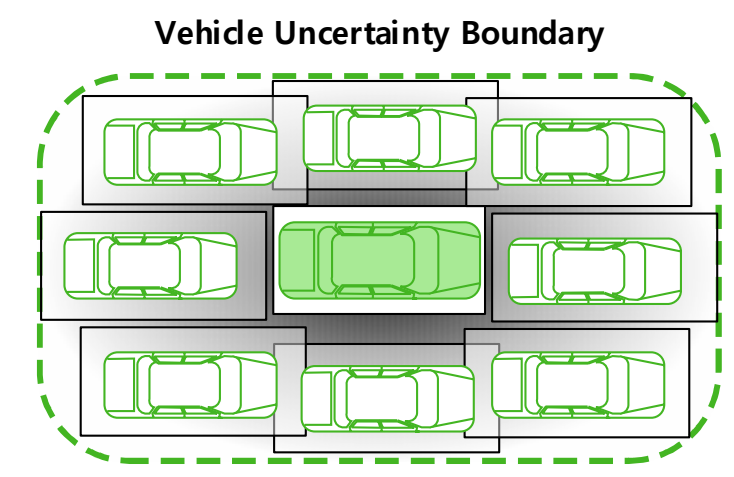

(b) Randomly Generated N State Vectors (Particles)

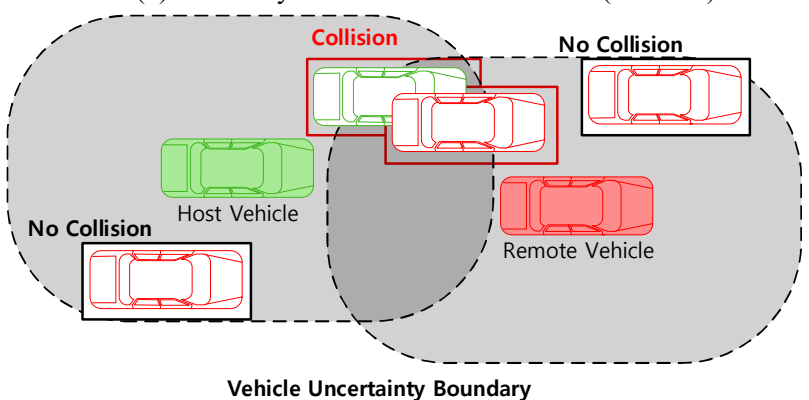

(c) Description of Collision

Fig.3. Procedure of Collision Probability Computation approximation accuracy. The state vectors are called particles and are denoted as (6) :

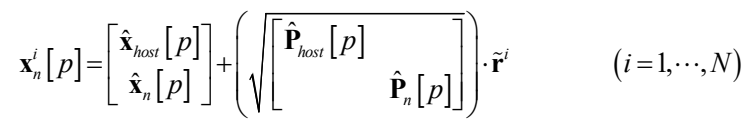

$\frac{\text { Input }}{\text { Predicted states and its covariance within a pre-defined }}$
prediction horizon for all tracked traffic participants.

For all tracked traffic participants, $n=1, \mathrm{~L}, N_{\text {target }}$

For every predictive time step, $p=0, \mathrm{~L}, N_{p}$

- Initialize collision count with participant $n$ at predictive time step $p$, C.P.cnt ${ }_{n}^{i}=0$.

- From the given predicted state and covariance, randomly generate $N$ particles, $\mathbf{x}_{n}^{i}[p]$.

For every particle, $i=1, \mathrm{~L}, N$

- Generate two vehicle-body-shapedpolygons.

- $\quad$ Check if the vehicle bodies can be possibly intersected.

- If intersection is detected,

$$
\text { C.P.cnt }{ }_{n}^{i}=\text { C.P. } \text { cnt }_{n}{ }_{n}+1
$$

- $\quad$ Else, C.P.cnt ${ }_{n}^{i}=$ C.P.cnt ${ }_{n}^{i}$.

\section{End}

- Approximate collision probability with participant $n$ at predictive time step $p$.

$$
\text { - } \quad \text { C.P. }{ }_{n}^{i}={ }^{2} \text { C.P.cnt }{ }_{n}^{i} / N \text {. }
$$

End End

where $p$ is the predictive time step, $\hat{\mathbf{x}}_{\text {host }}$ is the predicted position and orientation state of the host vehicle, $\hat{\mathbf{x}}_{n}$ is the predicted pose state of nth traffic participant (remote vehicle), $\hat{\mathbf{P}}$ denotes proper size of covariance matrix of each predicted state, and f/is a white noise random vector of proper size. For every possible pair of host and remote vehicle, we investigate whether the vehicle bodies of the traffic participant can intersect the host vehicle at each predicted time step.

The collision probability between the host and the remote vehicle can be computed by the number of collision case out of whole possible number of case that two vehicle randomly exist within uncertainty boundary. The collision case counts when two vehicles share a same part of the space as illustrated in Fig.3-(c). The uncertainty boundary in Fig.3-(b), can be modelled like stated in the previous section. The boundary, grey shaded ellipsoidal area, around the vehicle in Fig.3-(b) describes the reachable area.

For every random particle, vehicle-body-shaped-polygons of the host and remote vehicles are generated as indicated in Fig.3-(c). If randomly generated two vehicles' polygons share the same part of the space, the collision case is counted. As there are more number of space sharing area, collision case, the collision probability increases. Details of the algorithm are presented below.

\subsection{Active Safety Control Intervention Moment}

In order to guarantee the safety at any speed range, a humancentered risk assessment algorithm is implemented. Fig.4 expresses, the proposed human-centered active safety control algorithm is based on the peak (maximum) collision probability and the human 
reaction time with respect to most probable predicted collision distance. To obtain the human reaction time in terms of the most probable predicted collision distance, $\boldsymbol{D} \boldsymbol{p}_{\max } \boldsymbol{p}$, Table 1 describes the experimentally acquired and fitted data for the stopping sight distance with a range of speed.

The study of reaction times by ${ }^{(7)}$ was based on data 321 drivers who expected to apply their brakes. A $2.5 \mathrm{sec}$ of reaction time for stopping sight situations encompasses the capabilities of most drivers, including those of older drivers.

A stopping sight distance $(S S D)$ formula based on the design speed is derived like below.

$\operatorname{SSD}(t)=$ Driver perception - reaction distance + braking distance

$$
=0.278 \cdot v_{x}(t) \cdot T_{r}+1.075 \cdot \frac{v_{x}(t)^{2}}{a}
$$

$$
\begin{aligned}
& S S D=\text { required stopping sight distance }[\mathrm{m}] \\
& v_{x}=\text { Longitudinal Speed }[\mathrm{m} / \mathrm{s}] \\
& T_{r}=\text { brake reaction time }=2.5[\mathrm{sec}] \\
& a=\text { deceleration rate }=3.4\left[\mathrm{~m} / \mathrm{s}^{2}\right]
\end{aligned}
$$

Driver's deceleration magnitude is assumed to be $3.4 \mathrm{~m} / \mathrm{s}^{2}$. The driver deceleration results showed $3.4 \mathrm{~m} / \mathrm{s}^{2}$ as the $10^{\text {th }}$ percentile value; that is, when asked to stop as quickly as possible on wet pavements, most drivers selected decelerations of $3.4 \mathrm{~m} / \mathrm{s}^{2}$ or a bit greater $^{(7)}$.

The meaning of crossing point in Fig. 4 is the intervention moment of active safety control. In order to guarantee the robustness and safety of autonomous driving, it is important to find a best trade off between pure manual and fully autonomous driving

(8). In other words, the system has to find that moment between the human sense and automated function. The system status can be described by a pair of collision probability and predicted reaction time. And the function might be activated in anywhere in Fig.4, however, where to intervene is another issue. Unless the function intervenes at the proper moment, the driver could feel the system is too aggressive or too conservative ${ }^{(12)}$. By finding a trade off moment using general equilibrium analysis, driving efficiency, safety and driver acceptance can be achieved.

The intervention moment is found by using general equilibrium analysis ${ }^{(11)}$. According to this, right area of the crossing point can be decided it is safe because of enough reaction time, and the other

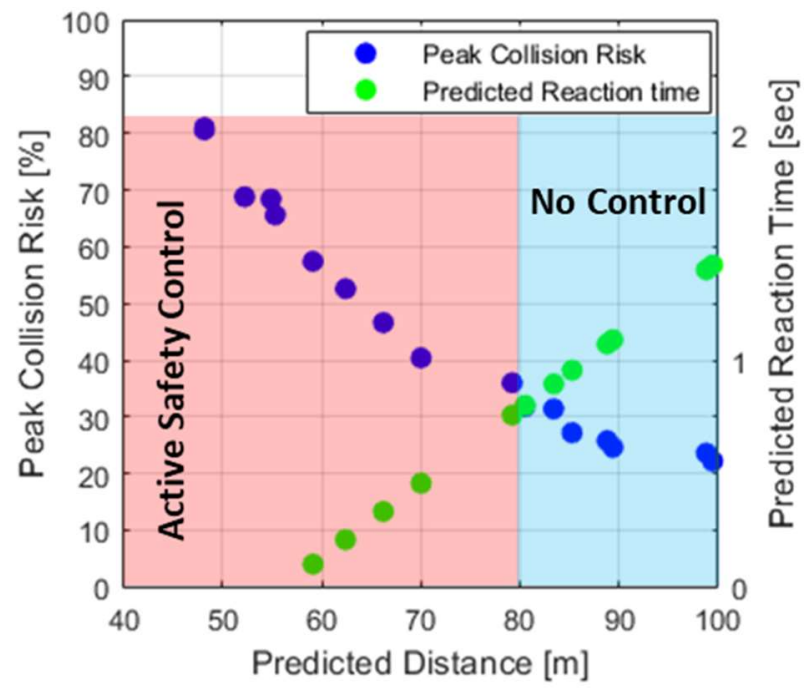

Fig.4 Human Centered Risk Assessment Algorithm
Table 1 Stopping Sight Distance for a Range of Speed

\begin{tabular}{cccc}
\hline \hline Speed $(\mathrm{km} / \mathrm{h})$ & $\begin{array}{c}\text { Brake } \\
\text { Reaction } \\
\text { Distance }(\mathrm{m})\end{array}$ & $\begin{array}{c}\text { Braking } \\
\text { Distance on } \\
\text { Level }(\mathrm{m})\end{array}$ & $\begin{array}{c}\text { Stopping } \\
\text { Sight } \\
\text { Distance }(\mathrm{m})\end{array}$ \\
\hline 20 & 13.9 & 4.6 & 18.5 \\
30 & 20.9 & 10.3 & 31.2 \\
40 & 27.8 & 18.4 & 46.2 \\
50 & 34.8 & 28.7 & 63.5 \\
60 & 41.7 & 41.3 & 83 \\
70 & 48.7 & 6.2 & 104.9 \\
80 & 55.6 & 73.4 & 129 \\
90 & 62.6 & 92.9 & 155.5 \\
100 & 69.5 & 114.7 & 184.2 \\
110 & 76.5 & 138.8 & 215.3 \\
120 & 83.4 & 165.2 & 248.6 \\
130 & 90.4 & 193.8 & 284.2 \\
\hline \hline
\end{tabular}

area is abnormal situation which has to be controlled by active safety function described in Fig.4.

The human sense can be expressed by human reaction time, which reflects human driver's ability. The automated system's performance can be described by collision probability in this paper. $2.5 \mathrm{sec}$ of reaction time describes the $100 \%$ of safe. The range of Predicted Reaction Time has been corrected to [0:2.5 sec] and this corresponds to the collision avoidance probability ${ }^{(13)}$. The $2.5 \mathrm{sec}$ of predicted reaction time is fixed since it reflects the collision avoidance capabilities of most drivers. In other words, it describes long enough reaction time for most drivers to avoid the collision with $100 \%$ of safety.

The simulation scenario in Fig. 4 is the preceding remote vehicle sudden braking scenario. In order to find the active safety control intervention point, a trade off moment, predicted reaction time, $R T p_{\max C p}$, which is rearranged from (7), can be computed

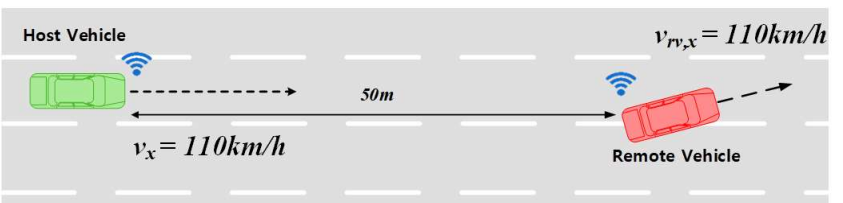

(a) Remote Vehicle Cut-in on the Highway

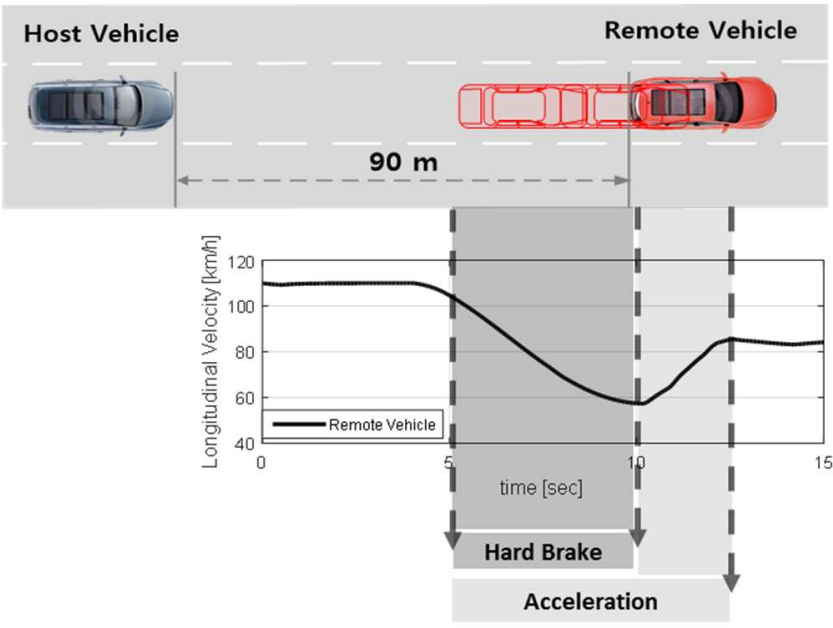

(b) Remote Vehicle Braking on the Highway

Fig.5 Simulation Scenarios 
according to the current host vehicle speed, $v_{x}$, and predicted stopping sight distance is $D p_{\max C p}$, as follows:

$R T p_{\max C p}(t)[\mathrm{sec}]=\frac{1}{0.278 v_{x}(t)}\left(D p_{\max C p}(t)-\frac{1.075 \cdot v_{x}(t)^{2}}{a}\right)$

Based on the active safety intervention moment in Fig.4, a crossing point, an intervention margin has been derived and it divides risk assessment plane as two areas. Before collision risk and reaction time are intersected together, blue area, it is safe (normal) driving mode. On the other hand, the red area means emergency or abnormal driving mode. The active safety control has to intervene at the crossing point which is a best trade off moment.

\section{Computer Simulation Studies}

To validate the effectiveness of the vehicle to vehicle wireless communication for application to automated vehicles, simulations are conducted on the Matlab/Simulink, connected to Carsim8.02. The simulations are based on the human-centered risk assessment algorithm in order to show the effectiveness of an inter vehicle communication. The remote vehicle state estimation performance has been verified via remote vehicle cut-in scenario in Fig.5-(a) by comparing three cases, i.e. real (Carsim), Radar only and the proposed V2V/Radar fusion. A vehicle control performance are investigated on the human-centered risk assessment algorithm with the scenario described in Fig.5-(b)

\subsection{Simulation Scenario}

Two simulation studies are investigated in this paper. The first is the simulation of the remote vehicle cut in on the highway. And the second study is the simulation of the response of the host vehicle to the hard deceleration of the preceding remote vehicle on the highway.

In the remote vehicle cut-in scenario, Fig.5-(a), initially a host vehicle is cruising at $110 \mathrm{kph}$. Then a vehicle in an adjacent lane cuts in front of the host vehicle with the 50 meters of initial longitudinal clearance. Without inter-vehicle communication, the host vehicle would detect the cut-in vehicle when the latter passes the lane border. The cut-in vehicle directly transmits yaw rate information to the host vehicle at the instant it starts to cut in from the center of the adjacent lane. According to Shin et al ${ }^{(2)}$. this means that the host vehicle will be able to have longer response time to slow down or respond with the aid of $\mathrm{V} 2 \mathrm{~V}$ communication.

The effect of communication on the response of the autonomously cruising host vehicle to the braking and accelerating of preceding remote vehicle is investigated. The scenarios are shown in Fig.5-(b). At the beginning, the host vehicle and the remote vehicle drive at $110 \mathrm{kph}$ with the range of 90 meters. The preceding remote vehicle starts sudden braking of $0.5 \mathrm{~g}$ at 4.8 seconds and then accelerates at 10 seconds. The following host vehicle is expected to autonomously apply a proper brake or acceleration to maintain the desired range.

In a conventional radar only using active safety scheme, onboard radar is used to measure the distance between the host vehicle and the remote vehicle, and the distance is numerically differentiated to get the relative velocity. With V2V communication, whenever the remote vehicle brakes, it directly transmits the deceleration information to the host vehicle. Shin et $\mathrm{al}^{(2)}$. investigated that the $\mathrm{V} 2 \mathrm{~V} / \mathrm{Radar}$ fusion case, upon receiving the message, brakes strongly enough for safety while ensuring driver's comfort.

\subsection{Remote vehicle dynamic state estimation performance}

Fig.6 illustrates the remote vehicle state estimation results for the remote vehicle cut-in scenario, Fig.6-(a), with sudden GPS

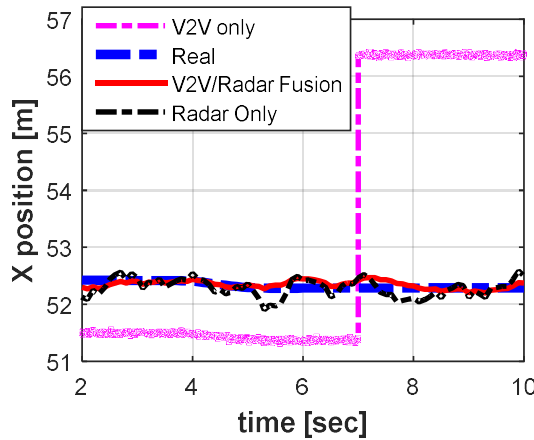

(a) Relative $\mathrm{X}$ position

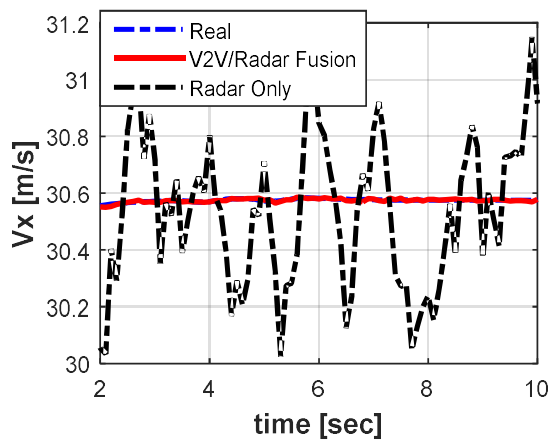

(d) Relative velocity

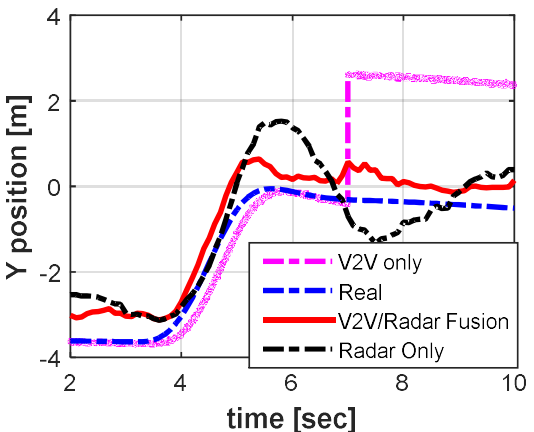

(b) Relative Y position

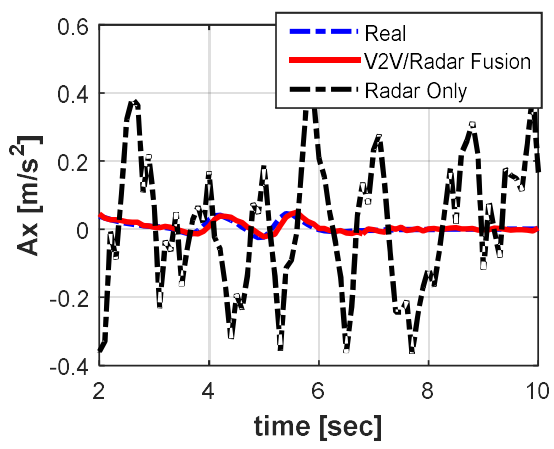

(e) Longitudinal Acceleration

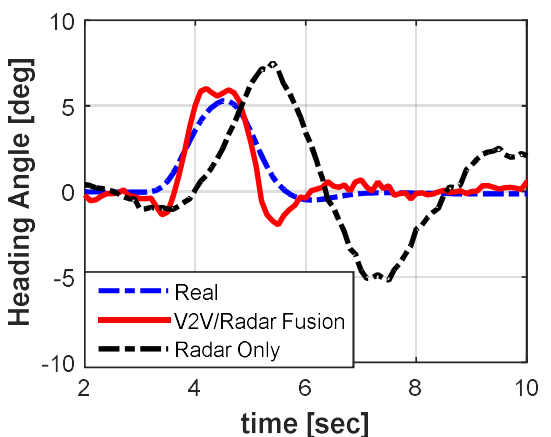

(c) Relative heading angle

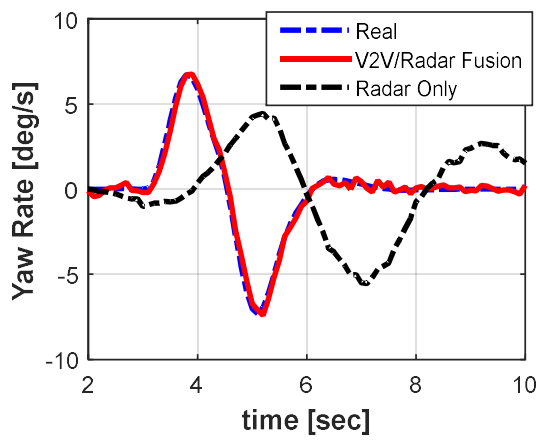

(f) Yaw rate

Fig.6. Remote vehicle state estimation results for the scenario in Fig.5-(a) 
errors of 3 to 5 meters from 7 seconds. The state estimation results in Fig.6 shows that V2V/Radar fusion algorithm performs really well even during when the GPS signal is poor or V2V communication delay exists. Since remote vehicle's precise CAN signals such as yaw rate and longitudinal acceleration are directly transmitted to the host vehicle, the host vehicle does not have to numerically compute the states of remote vehicle, and this results in significant improvement of the target object estimation performance.

\subsection{Effects of Vehicular Communication : Controlled Host Vehicle}

The human-centered risk assessment algorithm has been incorporated into a model predictive control (MPC) based longitudinal vehicle control in ${ }^{(10)}$. The objective of a longitudinal vehicle model is used and its objective is to maintain vehicle distance and adjust velocity to avoid the longitudinal collision. The state can be defined as three : clearance error, velocity error and longitudinal acceleration. And the control input is desired longitudinal acceleration derived from the longitudinal model. The effect of communication on the response of host vehicle to the braking of preceding remote vehicle is studied. The scenario is shown in Fig.5-(b). The remote vehicle applies a hard brake of approximately $0.4 \mathrm{~g}$ at 4.8 seconds and then accelerates again. As illustrated in Fig.7-(a), it can be seen that the V2V/Radar fusion vehicle brake response is 2 seconds earlier than the radar only case. Fig.7-(b) is the range, longitudinal clearance, between the host and the remote vehicle on the time domain. It can be clearly seen in Fig.7-(b) that the minimum range between two vehicles are extended by 14.24 meters because of V2V communication. Fig.7. shows that the simulation results confirm our belief that both collision risk and reaction time recover to safe region when the controller intervenes the system. Vehicular communication is of great benefit for the active safety control as indicated in Fig.8 -(a)

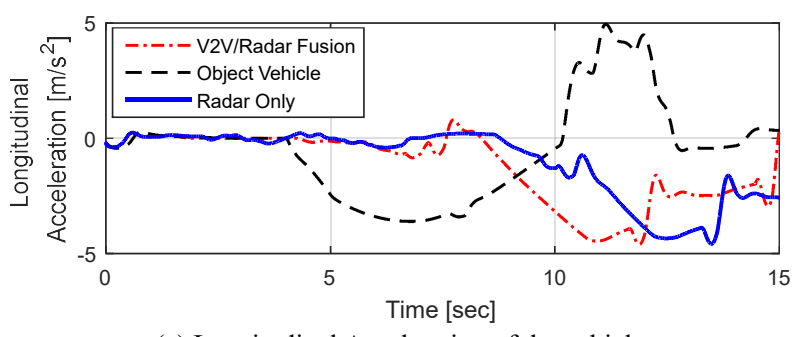

(a) Longitudinal Acceleration of the vehicles

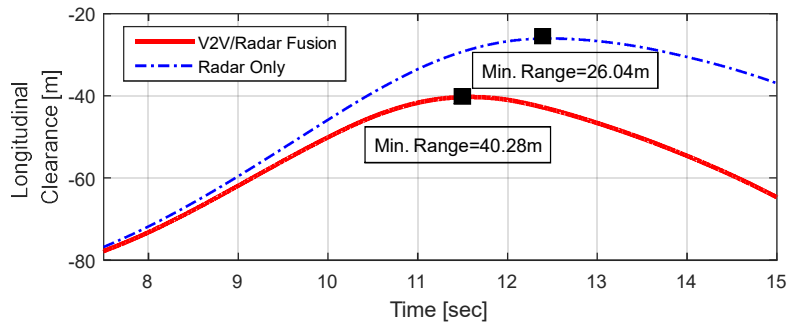

(b) Range of the vehicles

Fig.7 Simulation results for the host vehicle longitudinal control and (b). The longitudinal controller with radar only case does not fully guarantee the safety due to the fact that active safety intervention moment is 10 percent higher than its best intervention point. This means that predicted reaction time is only 0.1 second and predicted collision distance is 49 meters when the active safety intervenes the system.

However, simulation for the V2V/Radar fusion case in Fig.8-(a) expresses significant improvement in the risk assessment performance with the maximum 9 percent of peak collision risk difference. This results in faster intervention timing of controller

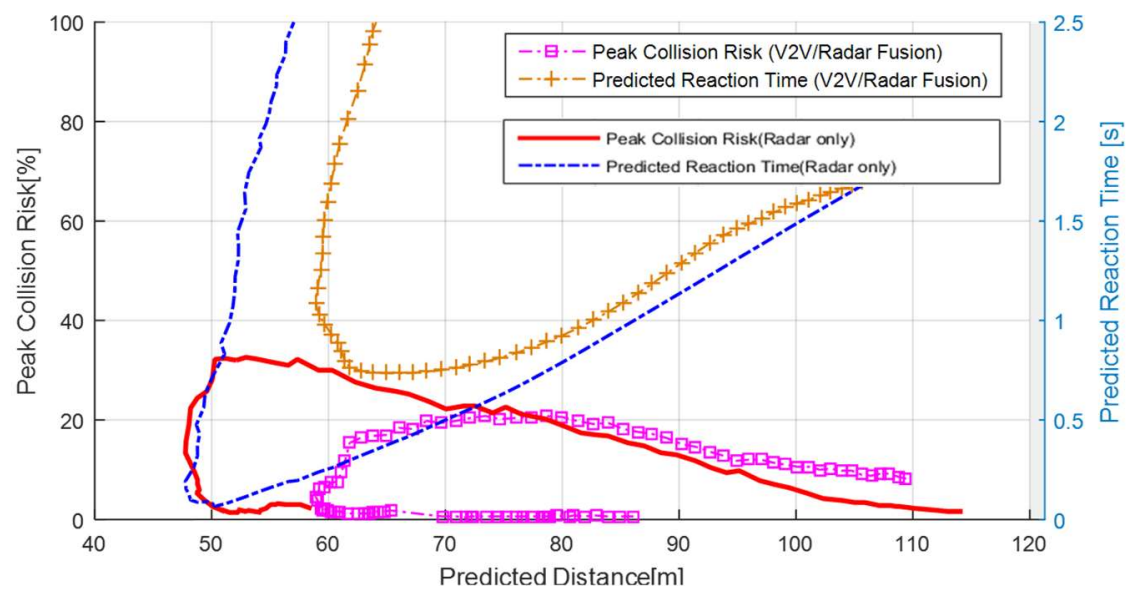

(a) Human Centered Risk Assessment: Combined View

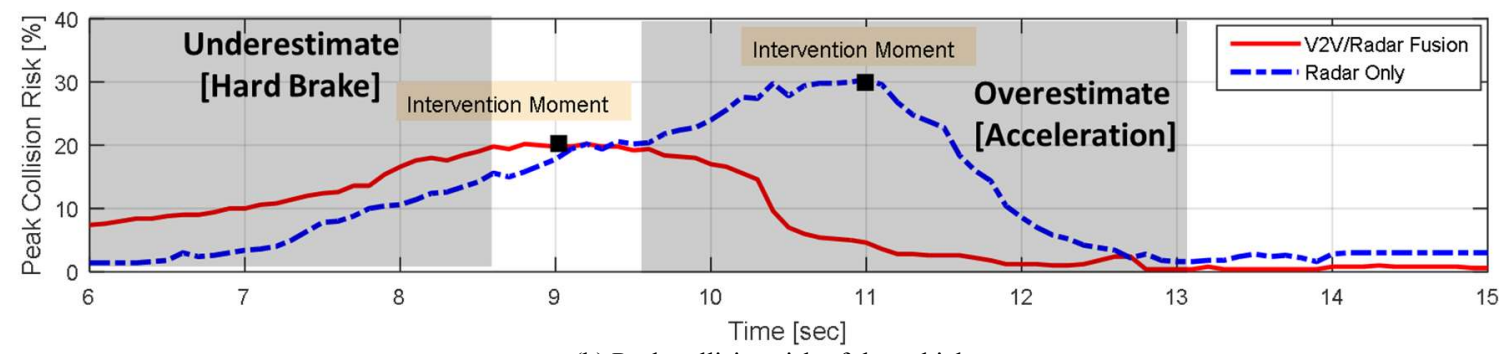

(b) Peak collision risk of the vehicles

Fig. 8 Simulation results for the host vehicle longitudinal control 
and the active safety intervention moment is 11 percent lower than radar only case. The collision risk when the active safety system intervenes is 19.4 percent and is no greater than a best trade pby 0.65 second and 10 meters, respectively. This means that it fully guarantees the safety compared to radar only case. Although a very conservative active safety system may be able to prevent all possible collisions, however, it will also be more likely disrupt the driver by applying the brakes at inappropriate times. It can be more improved by intervening the $\mathrm{s}$ ystem at a best trade off moment. The predicted reaction time and distance are extended by 0.65 second and 10 meters, respectively.

\section{Conclusion}

A human factor considered risk assessment of automated vehicle using vehicle to vehicle wireless communication has been presented. Although the information fusion of $\mathrm{V} 2 \mathrm{~V}$ communication and ADAS sensors has been disussed from many authors to date, however, we propose the realistic applications for risk assessment and collision avoidance through V2V communication. The proposed human-centered risk assessment algorithm can enhance the safety of vehicles using a track to track fusion algorithm. Using GNN approach, the target vehicle state estimation performances are greatly improved compared to radar only even during which the GPS signal is poor or theV2V communication delay exists. In order to decide active safety control intervention moment, a collision risk and a human reaction time are incorporated to achieve human like behavior of the automated vehicle. It was shown from both simulations that the active safety intervention decision is significantly enhanced with the V2V communication.

This paper is written based on a proceeding presented at JSAE FAST-zero'17 Meeting.

\section{Acknowledgement}

This research was jointly supported by the National Research Foundation of Korea(NRF) grant funded by the Ministry of Science, ICT and Future Planning (MSIP) (NRF-2016R1E1A1A01943543), and supported by the 2018 National Research Projects of Naval Research Center, Republic of Korea Naval Academy.

\section{References}

(1) D. Shin and K.Yi : Compensation of wireless communication delay for integrated risk management of automated vehicle, Proceedings of the IEEE International Conference on Intelligent Vehicle Symposium, pp. 1355-1360 (2015).

(2) D. Shin, B. Kim, J. Seo and K. Yi : Effects of Wireless Communication on Integrated Risk Management Based Automated Vehicle, Proceedings of the IEEE International Transportation Systems, pp. 1767-1772 (2015).

(3) T. J. Gordon and M. Lidberg : Automated driving and autonomous functions on road vehicles, Vehicle System Dynamics, Vol. 53, No. 7, pp. 958-994 (2015).

(4) B. Kim, K. Yi, H. J. Yoo, H. J. Chong and B. Ko : An IMM/EKF Approach for Enhanced Multitarget State Estimation for Application to Integrated Risk Management System, IEEE Transactions on Vehicular Technology, Vol. 64, No. 3, pp. 876-889, (2015).

(5) Laugier, Christian, Igor E. Paromtchik, Mathias Perrollaz, Mao Yong, John-David Yoder, Christopher Tay, Kamel Mekhnacha, and Amaury Nègre: Probabilistic analysis of dynamic scenes and collision risks assessment to improve driving safety, IEEE Intelligent Transportation Systems Magazine 3, No. 4, pp. 4-19, (2011).

(6) National Cooperative Highway Research Program (1997). NCHRP Report 400: Determination of Stopping Sight Distances (PDF). Transportation Research Board (National Academy Press). p. I-13. ISBN 0-309-06073-7.

(7) R. Layton : Stopping sight distance and decision sight distance, Research Institute Oregon State University, Corvallis, Oregon (1997).

(8) J. Wei and J. M. Dolan : A multi-level collaborative driving framework for autonomous vehicles, RO-MAN 2009 - The 18th IEEE International Symposium on Robot and Human Interactive Communication, Toyama, pp. 40-45 (2009).

(9) D. Shin, B. Kim and K. Yi : Probabilistic Threat Assessment of Vehicle States Using Wireless Communication for Application to Integrated Risk Management System," 2015 Proceedings of FAST-Zero'15, No.01602290, pp.235-242 (2015).

(10) J. Seo and K. Yi: Robust Mode Predictive Control for Lane Change of Automated Driving Vehicles," SAE Technical Paper, No. 2015-01-0317. (2015)

(11) Miller, Edward M. "Risk, uncertainty, and divergence of opinion." The Journal of finance Vol.32, No.4, pp.1151-1168. (1977)

(12) P. Seiler, B. Song and J. K. Hedrick:Development of a collision avoidance system, SAE Technical Paper, No. 980853. (1998)

(13) Sieber, Markus, and Berthold Färber: Driver perception and reaction in collision avoidance: Implications for ADAS development and testing, Proceedings of the IEEE International Conference on Intelligent Vehicle Symposium, pp. 239-245 (2016).

(14) S. Blackman and R. Popoli, Design and Analysis of Modern Tracking Systems. Norwood, MA: Artech House, 1999. 